\title{
Review of retinal cameras for global coverage of diabetic retinopathy screening
}

\author{
Ramachandran Rajalakshmi ${ }^{1}{ }^{1} \cdot$ Vijayaraghavan Prathiba $^{1} \cdot$ Subramanian Arulmalar ${ }^{1} \cdot$ Manoharan Usha ${ }^{1}$
}

Received: 2 September 2020 / Revised: 10 October 2020 / Accepted: 27 October 2020 / Published online: 9 November 2020

(c) The Author(s), under exclusive licence to The Royal College of Ophthalmologists 2020

\begin{abstract}
The global burden of diabetes has resulted in an increase in the prevalence of diabetic retinopathy (DR), a microvascular complication of diabetes. Lifelong repetitive screening for DR is essential for early detection and timely management to prevent visual impairment due to the silent sight-threatening disorder. Colour fundus photography (CFP) is helpful for documentation of the retinopathy as well as for counselling the patient. CFP has established roles in DR screening, detection, progression and monitoring of treatment response. DR screening programmes use validated mydriatic or non-mydriatic fundus cameras for retinal imaging and trained image graders identify referable DR. Smartphone-based fundus cameras and handheld fundus cameras that are cost-effective, portable and easy to handle in remote places are gaining popularity in recent years. The images captured with these low-cost devices can be immediately sent to trained ophthalmologists for grading of DR. Recent increase in numbers of telemedicine programmes based on imaging with digital fundus cameras and remote interpretation has facilitated larger population coverage of DR screening and timely referral of those with sight-threatening DR to ophthalmologists. Good-quality retinal imaging and accurate diagnosis are essential to reduce inappropriate referrals. Advances in digital imaging such as ultra-wide field imaging and multi-modal imaging have opened new avenues for assessing DR. Fundus cameras with integrated artificial intelligence (AI)-based automated algorithms can also provide instant DR diagnosis and reduce the burden of healthcare systems. We review the different types of fundus cameras currently used in DR screening and management around the world.
\end{abstract}

\section{Introduction}

Retinal imaging plays a significant role in the screening and management of diabetic retinopathy (DR), the most common microvascular ocular complication of diabetes. It is estimated that about 463 million people have diabetes worldwide, and if the same trend continues, this number is expected to increase to 578 million by 2030 and to an alarming 700 million people with diabetes by the year 2045 [1]. The rapid increase in prevalence of diabetes is expected to be higher in the low-to-middle income countries (LMIC). As previous global estimates have shown that one-third of the people with diabetes have some form of DR, this would

Ramachandran Rajalakshmi

drraj@drmohans.com

1 Department of Ophthalmology, Dr. Mohan's Diabetes Specialities Centre and Madras Diabetes Research Foundation, Chennai, India translate to 193 million people with some stage of DR by the year 2030 [2].

As DR is an asymptomatic condition that can progress to irreversible vision loss without timely treatment, it is a candidate condition that meets all the criteria for a universal screening programme [3]. It is one of the most common causes of avoidable blindness with diabetic macular oedema (DMO) and proliferative diabetic retinopathy (PDR) being the two major sight-threatening forms of DR (STDR) [3, 4]. Traditionally, ophthalmologists have screened for DR by performing direct ophthalmoscopy, slit lamp biomicroscopy and indirect ophthalmoscopy for entire retinal examination after pupil dilatation (mydriasis). As the clinical course of DR has a long asymptomatic phase, individuals with diabetes may not visit the ophthalmologist regularly for DR screening unless they are aware of visual loss due to STDR. A universal screening programme for DR aims early identification of people with DR who are at high risk and ensure timely referral to the ophthalmologist/ retina specialist to initiate treatment before the development of advance damage [3,5]. 
Fig. 1 Rural Teleophthalmology.

A Retinal imaging inside a mobile telemedicine van in Chunampet village, south India, performed by a local trained technician. B Retinal image with Topcon TRC NW200 nonmydriatic desktop fundus camera.
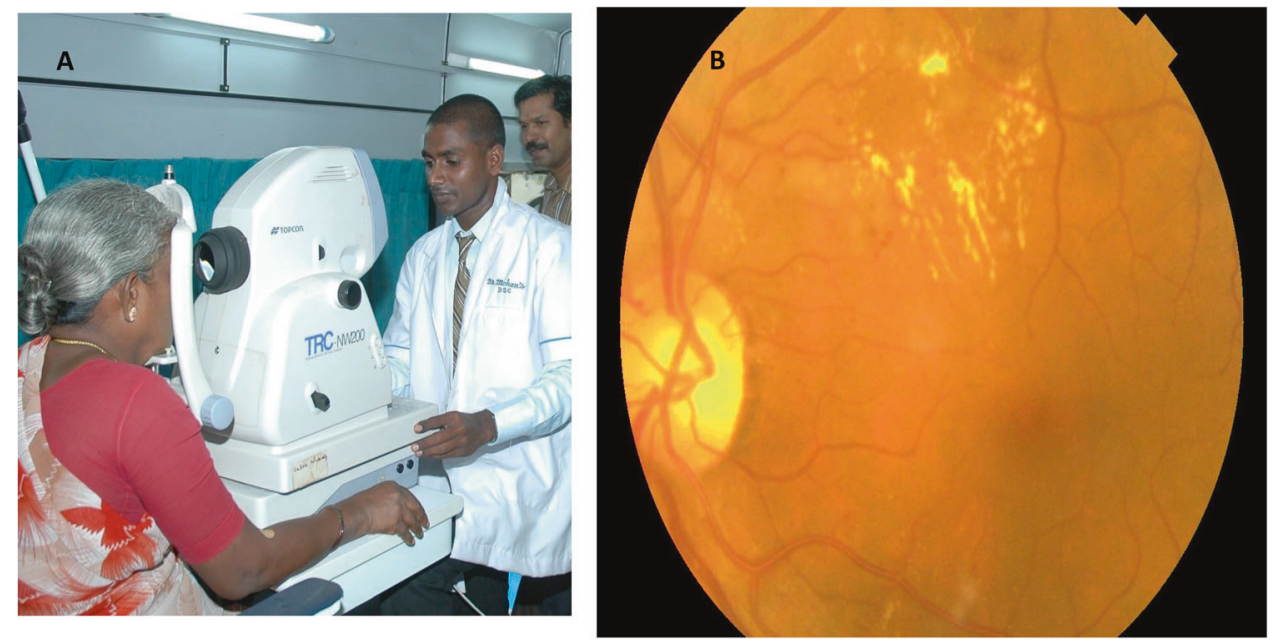

Lifelong evaluation for DR by repetitive retinal screening of people with diabetes is a valuable and essential strategy. Various imaging modalities, especially, colour fundus photography (CFP) has established important role in DR screening for early detection, assessment of progression and monitoring of treatment response. DR screening programmes employ different types of validated fundus cameras for retinal imaging and trained image graders/trained optometrists/ophthalmologists for interpretation of referable DR. Manual grading of fundus photographs for large numbers of people with diabetes by trained graders incur substantial costs to the healthcare systems. The advent of artificial intelligence (AI) and deep learning techniques has raised the possibility of automated DR detection-aided screening of DR [6].

This paper will review the different types of fundus cameras that are currently being used in DR screening and management around the world.

\section{CFP in DR screening and management}

Stereoscopic CFP of seven standard fields $\left(30^{\circ}\right)$ of the retina is the gold standard for detection of DR lesions as defined by the Early Treatment Diabetic Retinopathy Study (ETDRS) [7]. However, as this protocol is challenging to deliver in clinical practice, two or three field retinal images that capture $30^{\circ}-50^{\circ}$ of the posterior retina that includes the macula and the optic disc [8,9] are widely used to inform the DR status in ophthalmology clinics. Stereoscopic fundus imaging provides a pseudo three-dimensional image from an ordinary two-dimensional CFP by shifting the fundus camera a few millimetres between sequential photographs [9]. Some DR screening programmes use a single-field CFP but two or three field CFP provides better sensitivity and specificity in comparison $[8,10,11]$. The digital photographs may be interpreted by trained readers or forwarded to a reading centre for interpretation and grading of DR.

However, increasing prevalence of diabetes could soon globally outpace the supply of optometrists and ophthalmologists. In many LMIC, people have poor or even no access to ophthalmology care. In these settings, remote interpretation of retinal imaging may be employed. Screening of people with diabetes for early detection of DR should be feasible at their regular point of care for their diabetes such as at the diabetes clinics or primary care clinics. To achieve this, both mydriatic and non-mydriatic (NM) digital fundus photography are widely used in LMIC as practical alternatives for screening for DR [8, 10-12].

The main challenges for LMIC to replicate established DR screening programmes in high-income countries are the high cost of recommended fundus cameras, limited infrastructure, lack of trained personnel and easy accessibility to DR treatment. Remote digital retinal imaging via telemedicine provides a mechanism to extend eye-care services to underserved populations [13]. Technological advances in the development of fundus cameras have significantly improved retinal photography by trained non-qualified technicians, where local people in rural areas in LMIC are able to take good-quality retinal images with basic training. Figure 1 shows retinal imaging inside a mobile telemedicine van in Chunampet village, South India, performed by a local trained technician. Improvements in internet connectivity/telecommunication and smartphones are important breakthroughs that have made retinal imaging and DR screening in remote areas realisable through telemedicine programmes [13-15]. In 2004, the American Telemedicine Association provided guidelines and recommendations for clinical, technical and operational standards for DR screening that help address the barriers for DR screening [16]. In addition to DR, screening for ocular conditions such 


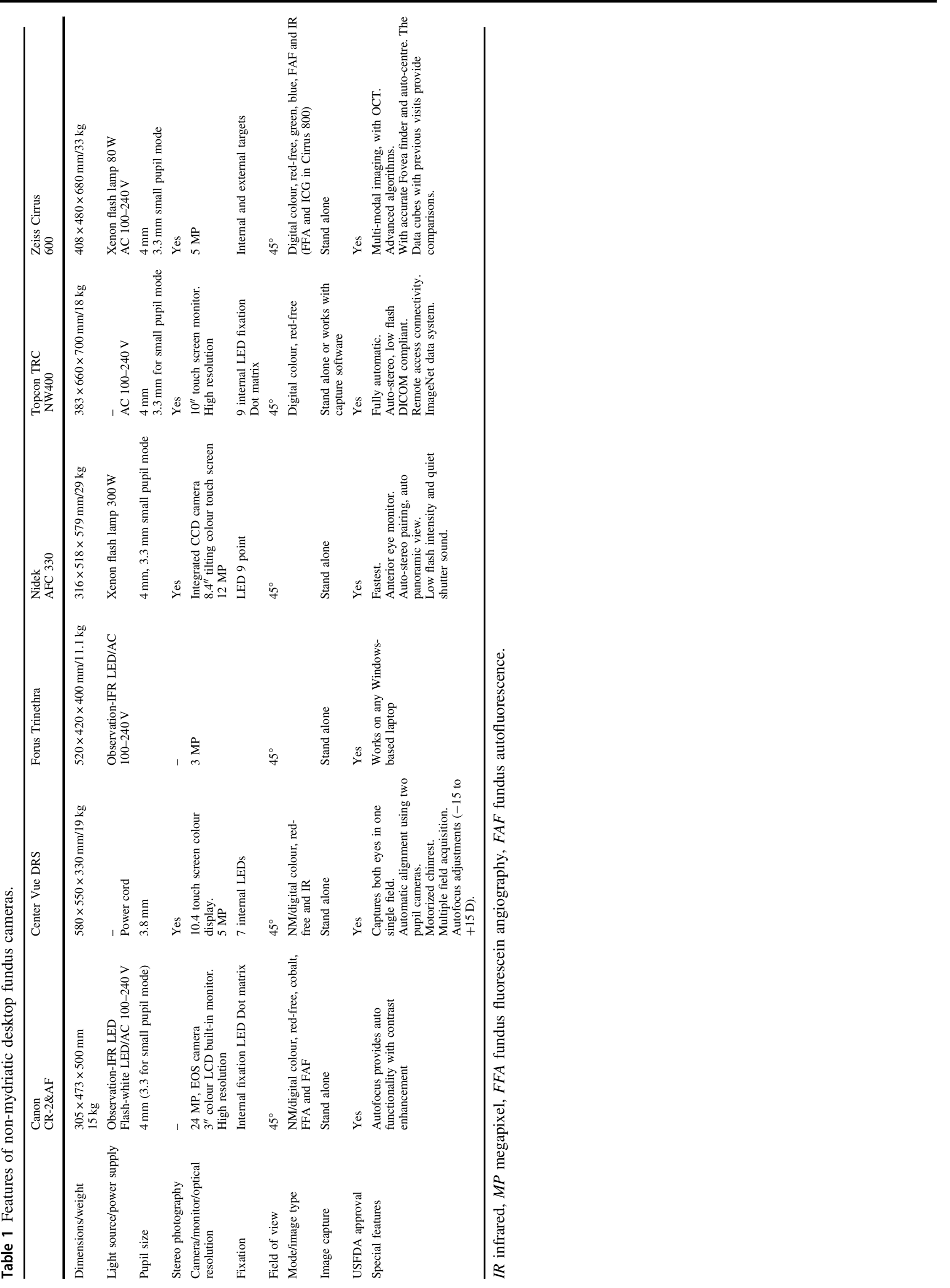


as cataract, hypertensive retinopathy, age-related macular degeneration (AMD) and glaucoma is also possible by retinal screening [17]. A full evaluation is required when a screening retinal photograph is ungradable.

\section{Advantages of digital retinal imaging in DR}

Digital imaging enables easy and immediate review of images, image magnification options with the ability to enhance and manipulate images. It provides scope for novel image analysis, and with internet connectivity, models of remote, system-assisted or automated AI-assisted diagnosis of DR is feasible. CFP is an effective tool to demonstrate clinical signs of DR and to show and educate the patient about their DR status. It is also useful for monitoring for improvement or progression of DR over time.

Fundus cameras used in DR screening can be conventional desktop cameras (mydriatic and NM), handheld cameras, smartphone-based fundus cameras and ultra-wide field (UWF) fundus cameras.

\section{Mydriatic desktop fundus cameras}

The classic conventional fundus cameras use $\times 2.5$ magnification with a $30^{\circ}$ field of view (FOV) (Zeiss FF, Carl Zeiss Meditec, Dublin, CA, USA). By taking stereo fundus images of various fields, it is possible to cover a wider region of the retina, including the optic disc, macula, vascular arcades and the region temporal to the macula, and can cover up to $75^{\circ} \mathrm{FOV}$. This formed the basis of the ETDRS grading system, where a 13-level DR severity scale was developed [7]. With multiple stereo images, the ophthalmologist or the certified grader has to evaluate each field, and then synthesise to provide an overall DR severity grade for each eye. This limitation can be overcome by combining the images into a montage. However, a montage fundus image may have artefacts or shadowing at the edges. The sensitivity and specificity for the detection of DR by ETDRS fundus photographic grading is superior to direct and indirect ophthalmoscopic examination by ophthalmologists. However, it requires an expensive desktop mydriatic fundus camera, trained certified retinal photographers and graders, and is labour intensive with a longer turnaround time.

Two-field mydriatic digital fundus photography performs well when compared to ophthalmoscopy and seven-field stereo fundus photography [10]. Mydriatic fundus imaging gives a sensitivity of $>80 \%$ in the detection of any grade of DR but caution has to be applied in the absence of ophthalmic professionals as there have been instances of mydriasis-induced angle closure glaucoma, and hence a sense of fear among physicians to use dilatation eye drops for mydriatic CFP. However, this is a rare complication and patients need to be advised to report to an ophthalmologist immediately if any symptoms occur. This should not deter the use of mydriatic fundus photography for DR screening.

\section{NM desktop fundus cameras}

NM fundus cameras (NMFC) have been used for evaluating DR for the last 25 years [18, 19]. In the USA and Europe, regular screening of DR using NMFC is being widely recommended [20, 21]. However, in India, studies have shown that large number of poor-quality ungradable photographs and low sensitivity limit the use of NMFC as a perfect screening system, particularly in Indian eyes with dark iris [22].

Two-field NM fundus photography is a useful tool in DR screening in the primary healthcare settings or for mass screening in camps. In NMFC, the reflective property of the retina is used. Majority of the NMFC have useful features including $45^{\circ} \mathrm{FOV}$, autofocus, auto-alignment, user interphase software, task automation, storage of images, printing images and export of images to a database and sharing retinal images through a network or internet connection. There are many NM desktop fundus cameras available in the market. The comparison of a few NMFC is shown in Table 1. For NM imaging, the room has to be minimally illuminated to aid physiological mydriasis. Infrared (IR) light is used in NMFC as it does not stimulate the pupillary contraction and also helps in alignment and focussing. Flash is used for image capture and low flash setting helps to minimise the persistent pupillary contraction.

NMFC CFP is useful in DR screening in the primary healthcare setting due to their ease of use, being patient friendly as the wait for dilatation is not required, better patient comfort as no bright flash of light is used, useful in people with shallow anterior chamber or narrow angles for whom dilatation is contraindicated [20]. The cameras can be operated by trained technicians and does not require skilled optometrists.

Several studies have examined the sensitivity and specificity of NM digital imaging [8, 19-24]. Retinal photography with NMFC has been found to be more sensitive than direct ophthalmoscopy in the detection of DR [19]. Other studies have shown NM CFP to be at least as efficient as direct ophthalmoscopy at screening for DR [23] and single-field $45^{\circ} \mathrm{NM}$ monochromatic images highly correlated $(\kappa=0.97, p=0.0001)$ to the gold standard stereoscopic 7-field mydriatic images [24]. Another study that compared single field versus 3-field NM images compared to the 7-field gold standard concluded that 3-field NM retinal images had a sensitivity and specificity of $82 \%$ and $92 \%$, respectively [8]. The AAO meta-analysis determined that single-field photography with NMFC can serve as a 
Fig. 2 Retinal imaging with hand-held fundus camera. A Retinal imaging with Zeiss Visuscout 100 handheld fundus camera. B Retinal image of sight-threatening diabetic retinopathy with post laser status centre involving diabetic macular oedema taken with Visuscout camera.
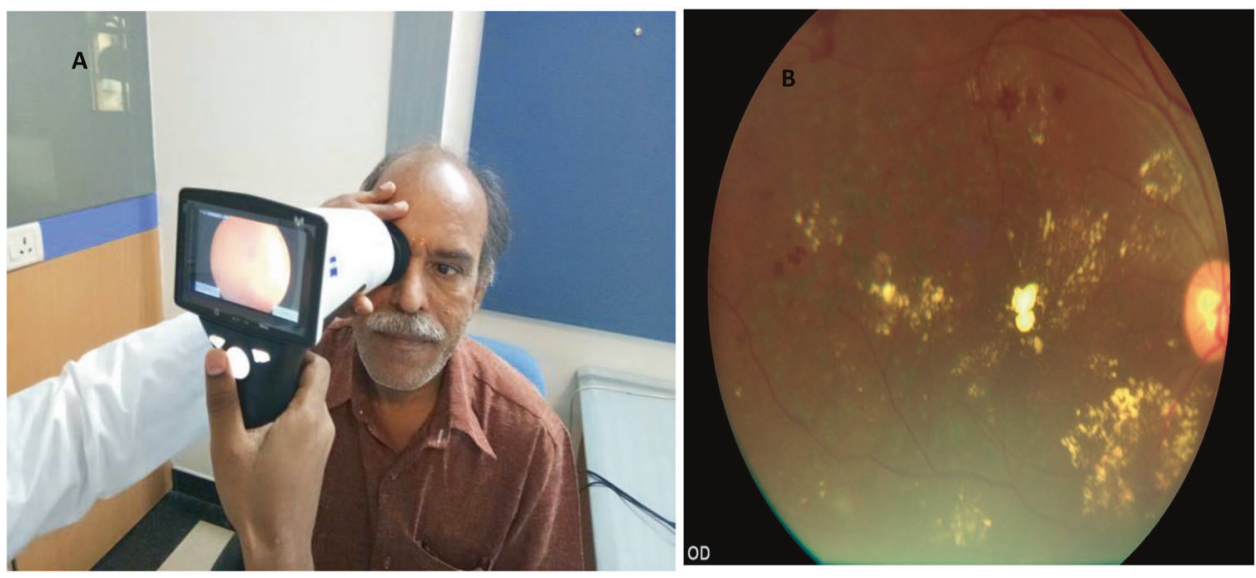

screening tool for DR to identify people with STDR for referral $[21,24]$.

NM fundus imaging has emerged as a popular tool for screening DR, but has limitations including a higher technical failure rate resulting from small pupils, especially in people with dark iris and media opacities like cataract in countries like India [22]. A study by Murgatroyd et al. showed that in retinal imaging with NMFC, mydriasis reduced the proportion of ungradable photographs from 26 to $5 \%$ [25].

Stereo fundus photography is also possible with NMFC. Hybrid cameras have features of both mydriatic and NMFC [26]. These hybrid instruments use the NM mode to capture CFP and mydriatic mode for people with hazy media and for fluorescein angiography (FFA) and fundus autofluorescence (FAF). The Canon CX-1 Hybrid Digital mydriatic/NM retinal camera, Topcon TRC NW8-F Plus, Zeiss Cirrus Photo are examples of hybrid cameras that, with the push of the myd/non-myd button, can select five photography modes, including colour, red-free, cobalt, FFA and FAF.

\section{Handheld fundus cameras}

Handheld digital cameras are portable, require less space, minimum power consumption and less skills and training [27]. The use of handheld retinal cameras for systemic DR screening in various settings and outcomes mainly depend on the image quality [28]. An earlier study done comparing Nidek NM-100 handheld camera showed poor image quality with the handheld camera when compared to Zeiss desktop camera [29]. Now with more technological advances, there are many validated handheld portable NM cameras that are currently available: Zeiss Visuscout 100 (Carl Zeiss, Jena, Germany), Smartscope Pro (Optomed, Oulu, Finland) Volk Pictor Plus (Volk Optical, Mentor, OH, USA), VersaCam TM DS-10 (Nidek, Gamagori, Japan), Horus DEC 200 (MiiS, Hsinchu, Taiwan) and Genesis-D
(Kowa, Nagoya, Japan), and are being used for DR screening, some of which are discussed below.

\section{Zeiss Visuscout 100}

This handheld imaging system has the ability to capture colour and red-free retinal images in a range of +20 to -20 $\mathrm{D}$ with $40^{\circ} \mathrm{FOV}$. The camera comprises of nine fixation targets and resolution of the camera is 5 megapixels (MP). Piyasena et al. compared fundus images taken pre and post dilatation with Visuscout 100 and graded by physicians against clinical examination by ophthalmologist using mydriatic bio-microscopy [28]. $43.4 \%$ of retinal images were found to be ungradable with Visuscout NM camera, which decreased to $12.8 \%$ after pupillary dilatation. Results showed that for referable DR, the sensitivity was $88.7 \%$ for grader 1 (positive predictive value [PPV] 59.1\%) and 92.5\% for grader 2 (PPV 68\%), using mydriatic imaging. The Ornate India study SMART India study is an on-going study in India that is using the Visuscout handheld camera for retinal imaging for DR screening in the community in 20 regions in India and is expected to recruit over 10,000 people with diabetes [30]. Figure 2 shows retinal imaging with Visuscout fundus camera.

\section{Optomed Aurora/Smartscope}

This handheld digital NMFC has a retinal module and an anterior module with a $50^{\circ} \mathrm{FOV}$ and a 5-MP resolution [31]. A study was done using Smartscope (the previous version of Optomed) handheld NMFC for screening of STDR compared with Topcon desktop mydriatic camera [32]. It concluded that out of 2475 images, $76.2 \%$ of Smartscope NM images, $90.1 \%$ of Smartscope mydriatic images and $92.0 \%$ of Topcon mydriatic images were gradable. Optomed Aurora has a wireless local area network module, which enables wireless data transfer to a computer. 


\section{Volk Pictor Plus}

Results of a prospective study done with Pictor Plus handheld NM camera with $40^{\circ} \mathrm{FOV}$ in individuals with diabetes showed that $86-94 \%$ of the retinal images were gradable pre-dilation and $94-97 \%$ of the images were gradable post dilatation [33]. The overall sensitivity for STDR was $64-88 \%$ and specificity was $71-90 \%$ as compared to the dilated fundus examination. The authors concluded that this handheld camera could capture retinal images of sufficient quality to screen for DR with and without dilation [33]. The device also has a dermatoscopic and otoscopic module making it very versatile and useful for clinicians in a multi-speciality setup.

The other handheld portable fundus cameras available are the Bosch camera, which has separate NM and mydriatic modes with $40^{\circ} \mathrm{FOV}$, and Signal handheld NM camera by Topcon, a new ultralight weight NMFC that provides a $40-50^{\circ}$ FOV with autofocus and manual focus $(-20$ to +20 D) options. The nine fixation targets offer both central as well as peripheral imaging. Handheld retinal cameras require more eye-hand coordination and alignment [34]. The advantages of handheld cameras are the lower cost and light weight but proper validation studies should be undertaken to assess the image quality before regular use in DR screening programmes $[34,35]$. Facilities to refer those with ungradable retinal images should be also available to help in detection of STDR.

\section{Smartphone-based fundus cameras}

Fundus imaging utilising the smartphone cameras along with the adaptor and the software has become an alternative mode of retinal imaging. Smartphones can be used with or without adaptors to capture retinal images.

Smartphones can be used like an indirect ophthalmoscope using the camera in the smartphone in video mode, with flashlight on and positioning the 20-D lens in front of the patient's eye to obtain fundus images. The "Comparison Among Methods of Retinopathy Assessment" Study compared smartphone-based fundus photography with 20-D lens, NM fundus photography and 7-field mydriatic fundus photography for DR detection and grading. Smartphone fundus photography with 20-D lens showed 59\% sensitivity and $100 \%$ specificity in detecting STDR [36].

Some models of smartphone-based cameras are currently available where fundus images can be taken directly from a smartphone, using a small compact adaptor: Peek Retina (Nesta, London, UK), PanOptic + iExaminer (Welch Allyn, Skaneateles Falls, NY) and D-Eye (D-EYE, Padova, Italy). These compact adaptor-based devices have some drawbacks: imaging with Peek Vision devices is possible after mydriasis, and PanOptic and D-Eye have very limited FOV $\left(25^{\circ}\right.$ and $20^{\circ}$, respectively).
D-Eye is an adaptor compatible with both iPhone and android smartphones and is approved by United States Food and Drug Administration (USFDA) and the European Union CE. The D-eye lens uses the camera and the LED light source of the smartphone for NM retinal imaging tool. It has an iOS-based operating system and an app for image storage with a working distance of $1 \mathrm{~cm}$ with autofocus between -10 to $+5 \mathrm{D}$ with a $5-8^{\circ} \mathrm{FOV}$ in undilated eye and $20^{\circ}$ FOV for dilated eye. Studies done on D-Eye have shown considerable agreement in grading of DR with $81 \%$ sensitivity and $98 \%$ specificity for detecting clinically significant macular oedema [37]. Optic nerve head (ONH) evaluation using smartphone-based retinal imaging is promising and dilation improves the image quality [38].

The iExaminer is an USFDA-approved adaptor that fits on the Panoptic Ophthalmoscope and aligns the iPhone camera lens with the Panoptic's viewfinder. The iExaminer can function as a NMFC with a $25^{\circ} \mathrm{FOV}$. The restricted FOV is the main disadvantage compared to other adaptors.

Peek Retina has an adaptor clip with a prism to deflect the light from the camera flash. It can be slipped into the camera of the smartphone that autofocuses for retinal imaging, and the Peek app helps in data and retinal image acquisition and storage. ONH images of Peek Retina were comparable to those acquired through desktop retinal camera [39].

There are other validated smartphone-based imaging systems with larger adaptors that are now commercially available like the Remidio Fundus on Phone (FOP) (Remidio Innovative Solutions, Bangalore, India), Volk iNview and Vistaview (Volk Optical, Mentor, OH, USA), Cellscope Retina and Make In India Retinal Camera (MII Retcam).

\section{FOP camera}

FOP is a smartphone-based, USFDA-approved validated retinal imaging system. It has various modules available currently including the mydriatic (M), NM and anterior imaging module. Patient data can be entered in the app and retinal imaging can be done in the camera with autofocus of the smartphone. The images can be stored in a folder in the smartphone or in iCloud and can even be sent to an external server for telemedicine. The customised report with fundus images can be printed immediately using a WiFi printer.

FOP-M camera has an annular illumination optical design, which can be used along with smartphones for acquiring and transmitting retinal images. It has a $45^{\circ} \mathrm{FOV}$ with \pm 20 -D adjustment with $\times 12$ optical magnification [40]. Validation studies of retinal imaging with FOP-M camera for DR screening in comparison with conventional mydriatic desktop fundus camera have shown a high sensitivity and specificity for detection of any grade of DR and 
Fig. 3 Comparison of retinal imaging between conventional fundus camera and smartphone based fundus camera. A Fundus image of branch retinal vein occlusion in conventional desktop camera. B Fundus image of branch retinal vein occlusion in smartphonebased Fundus on Phone (FOP) mydriatic camera.
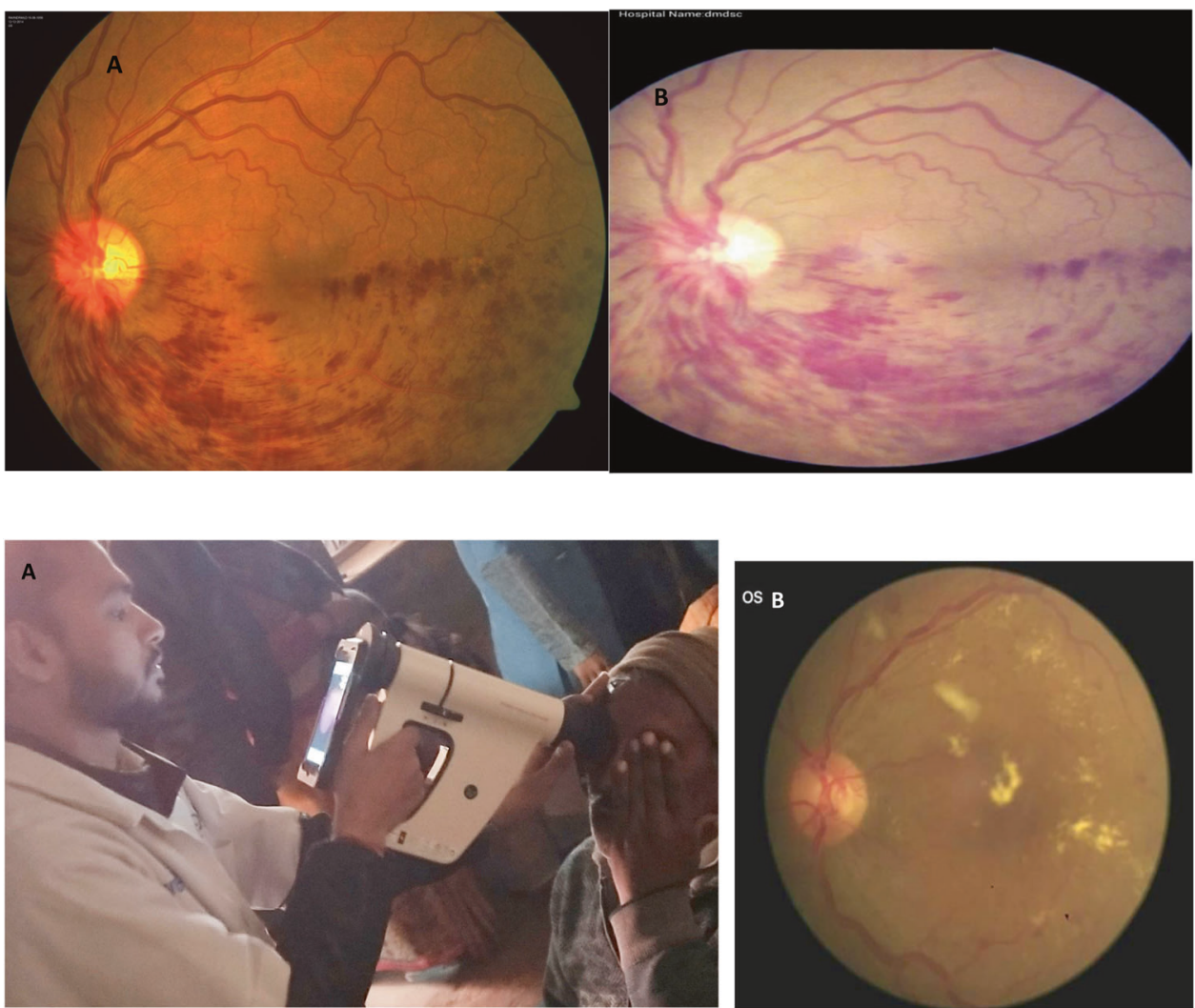

Fig. 4 Diabetic retinopathy screening in camps. A Retinal imaging with Fundus on Phone (FOP) non-mydriatic (NM) smartphone-based camera in a DR screening camp. B Retinal image of sight-threatening diabetic retinopathy taken with FOP NM camera.
STDR [40, 41]. Figure 3 shows fundus imaging with conventional camera and FOP-M camera.

FOP NM uses an iPhone with IR light for NM fundus imaging. The device has $40^{\circ} \mathrm{FOV}$ and $\times 10$ magnification with a built-in smartphone app with picture storage, retrieval and cloud sync functions. Advanced options like image montaging, glaucoma screening assist and AI-based image analysis for automated DR screening are available. The device can be used both in tabletop mode with a chinrest, fixed to a slit lamp or used in a handheld mode. Figure 4 shows FOP NM camera being used in the handheld mode for DR screening in a camp. A recent clinic-based study done in 245 participants with diabetes by Prathiba et al. showed that FOP NM cameras had high sensitivity and specificity for DR and STDR detection when compared to gold standard conventional desktop camera [42]. Studies have also shown high sensitivity and specificity of AIassisted detection of DR and STDR with FOP images [43, 44]. Currently FOP is being used at various Dr Mohan's Diabetes Specialities Centre diabetes clinics across India for tele-ophthalmology DR screening [13].

\section{Volk iNview and Vistaview}

The Volk iNview is an ophthalmic camera device, which is able to capture fundus images using an Apple iPhone or
iPod with images of 1-MP resolution with a static $50^{\circ} \mathrm{FOV}$. It has an inbuilt app where patient data can be entered, and fundus images can be stored in HIPAA-compliant storage and images can be exported to a computer.

Vistaview is a mydriatic smartphone fundus camera that weighs $500 \mathrm{~g}$, with auto and manual focus features, provides a $55^{\circ} \mathrm{FOV}, 3072 \times 2122$ pixels resolution with special features of voice-activated retinal image capture.

\section{Cellscope Retina}

Cellscope Retina is a handheld 3D-printed optical and hardware automated smartphone-based retinal imaging system with inbuilt app capable of capturing high-quality, wide-field images of the retina and weighs only $310 \mathrm{~g}$. The images can be uploaded to a secure server through the app. It provides a 5 image montage with a $100^{\circ} \mathrm{FOV}$ with 52.3 pixel per retinal degree resolution. Cellscope Retina had a good sensitivity and moderate specificity for referable DR [45].

\section{Make In India Retinal Camera (MII Retcam)}

MII Retcam device can image both posterior pole and the peripheral retina. The device has slots for both the smartphone and a 20-D lens as shown in Fig. 5. It works 
Fig. 5 A MII RetCam. B Retinal image of sight-threatening diabetic retinopathy taken with MII Retcam.
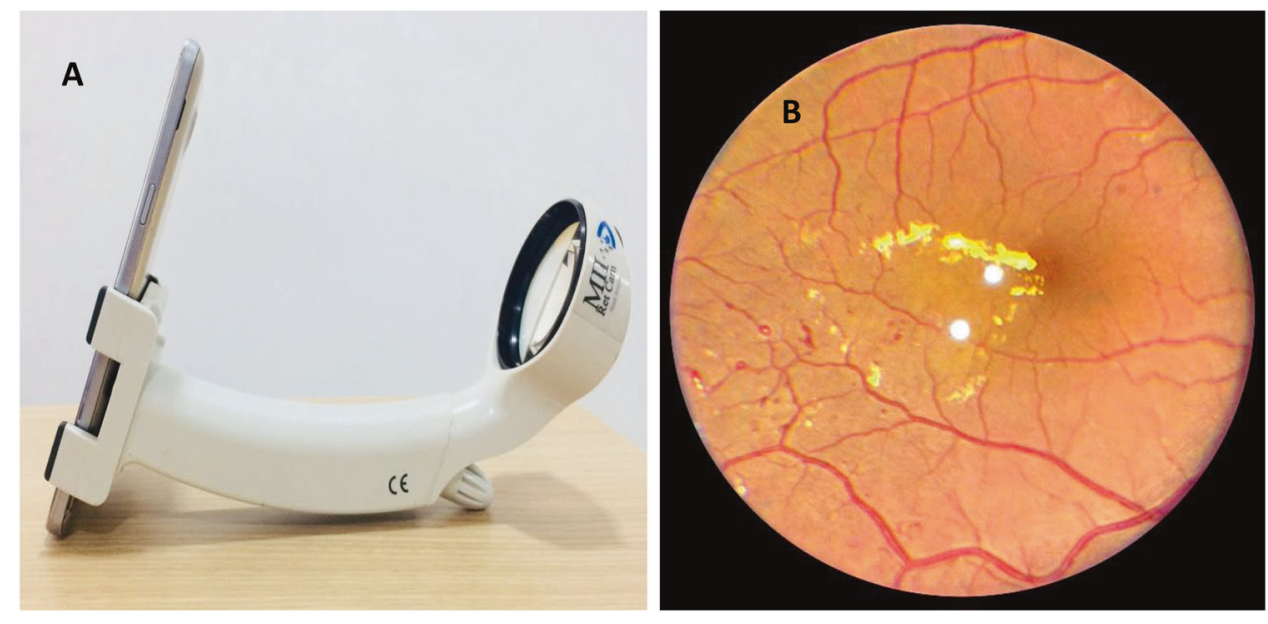

as a fundus camera and provides retinal videos and still images, has an application for patient data, image storage and retrieval. It is portable and can be used as a screening device for DR and retinopathy of prematurity [46].

\section{UWF fundus cameras}

In the recent years, UWF imaging, allows unprecedented view of the posterior pole. UWF imaging can provide up to $100-200^{\circ} \mathrm{FOV}$ of the retina allowing simultaneous evaluation of the peripheral and central retina. The UWF fundus camera uses a confocal scanning laser ophthalmoscope (CSLO) with an ellipsoid mirror to view $200^{\circ}$ of retina, which is $82 \%$ of retina as compared to normal fundus cameras, which view $30-50^{\circ}$ of retina. CSLO uses a laser light and forms an image by a detailed scanning of the entire field and then capturing the reflected light through a small confocal pinhole. The advantages include improved image quality, patient comfort, threedimensional imaging and video capability and effective imaging of patients who do not dilate well. Advent of UWF imaging using newer cameras such as Pomerantzeff camera, Retcam (Clarity Medical Systems, Inc., Pleasanton, CA, USA), ZEISS Clarus 500 (Zeiss, Carl Zeiss Meditech, Inc., Dublin, USA) Optos Optomap/Daytona Plus camera (Optos PLC, Dunfermline, UK) and the Staurenghi lens (Ocular Staurenghi 230 SLO Retina Lens; Ocular Instruments Inc, Bellevue, WA, USA) have made a radical change in the way we view the fundus [47].

Table 2 shows the different features of some UWF retinal cameras. The Optos Optomap is based on a CSLO with two laser frequencies coupled with a parabolic mirror that allows sampling of the $82 \%$ of the retinal surface with a single acquisition $\left(200^{\circ} \mathrm{FOV}\right)$ without dilation. The CFP in Optos Optomap relies on pseudo-colours, i.e., fake colours derived from the combination of red and green colour lasers while the UWF CFP in Zeiss Clarus uses true colours. Figure 6 shows a UWF fundus image of PDR with fresh pre-retinal haemorrhage in an individual who was allergic to dilating eye drops.

The DR lesions that are detected in the retinal periphery have the potential to change the grading of disease severity and may be of prognostic significance to disease progression. Studies have shown that UWF fundus photography has a high sensitivity (99\%) and specificity (100\%) in detecting DR [48]. Many DR lesions, intraretinal haemorrhages, microaneurysms, intraretinal microvascular abnormalities and new vessels elsewhere were located predominantly outside the standard seven fields. They were associated with increased risk of progression in 4 years irrespective of severity of DR and HbAlc levels [49]. Studies have shown that peripheral ischemia could be related to the presence and severity of DMO [50]. Silva et al. reported that the use of UWF CFP significantly increased identification of DR and STDR compared to NM fundus photography [50]. The retinal image acquisition time using UWF cameras is significantly shorter compared to ETDRS 7-standard CFP [51]. UWF fundus imaging is slowly getting considered as the gold standard for DR screening in the United States. However, it is an expensive modality for screening DR, especially for consideration in use in LMIC.

\section{Other newer DR imaging screening options}

\section{Fundus autofluorescence (FAF)}

FAF is a non-invasive retinal imaging, which identifies the autofluorescence of lipofuscin present in the retinal pigment epithelium (RPE). Multiple commercially available imaging systems, including the newer desktop fundus cameras, the CSLO UWF fundus cameras, are available to 


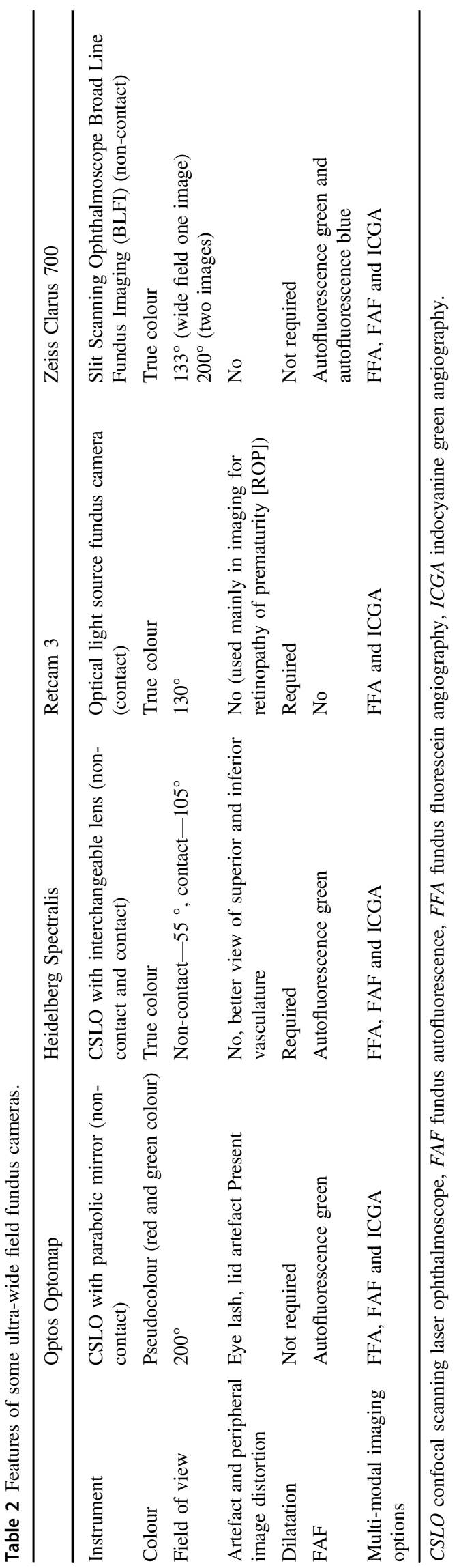

assess FAF. The FAF mode helps monitor macular waste such as lipofuscin, which assists with detecting AMD, macular dystrophies and DR. In normal individuals the autofluorescence of RPE layer is masked by the lutein pigment at the fovea, whereas in individuals with diabetes with cystoid macular oedema (CMO), they were seen as round or oval areas of autofluorescence at the fovea. FAF has shown $81 \%$ sensitivity and $69 \%$ specificity when compared with standard FFA in diagnosing CMO [52]. The role of FAF in DR screening remains to be understood.

\section{Multicolour imaging}

Reports have shown that multicolour fundus imaging is superior to traditional CFP in detecting and localising foveal cysts, and helps in better delineation of clinical signs of DR [53]. The Heidelberg Spectralis CSLO has a multicolour imaging system that acquires reflectance at three different wavelengths; blue, green and IR, that are superimposed to provide diagnostic information from different layers of the retina [53]. Multicolour imaging is a potential tool for screening DR in addition to CFP, though it is much more expensive [54].

\section{Conclusions}

The advances in digital fundus cameras have facilitated rapid acquisition and interpretation of fundus images and the rapid deployment of retinal imaging for DR screening worldwide. Telecommunication technology along with use of portable, easy-to-operate, less expensive validated fundus cameras have made cost-effective DR screening possible, even in remote villages. Effective screening is a desirable method for the early detection and successful treatment for $\mathrm{DR}$, and CFP is currently the main retinal imaging used for screening. Affordability, accessibility and accuracy are essential aspects in mass DR screening programme especially in LMIC. Improvements in retinal imaging and diagnostic accuracy are essential to reduce inappropriate referrals to retina specialists. In the recent years, UWF imaging, allows unprecedented view of the entire retina. Use of sleek devices (smartphone-based fundus cameras/ handheld fundus cameras), UWF fundus imaging and AIbased automated diagnosis are likely to become essential aspects of screening worldwide with the increasing prevalence of DR. Automated retinal image analysis platforms appear promising and can potentially reduce the burden on human graders. Advance fundus imaging with CSLO, multicolour imaging and multi-modal imaging could lead to more efficient screening with the possibilities of their routine use in DR screening in the future. 
Fig. 6 Wide field retinal imaging in diabetic

retinopathy. Ultra-wide field fundus image of post panretinal laser photocoagulation proliferative diabetic retinopathy with fresh pre-retinal haemorrhage taken with Optos Daytona plus camera.

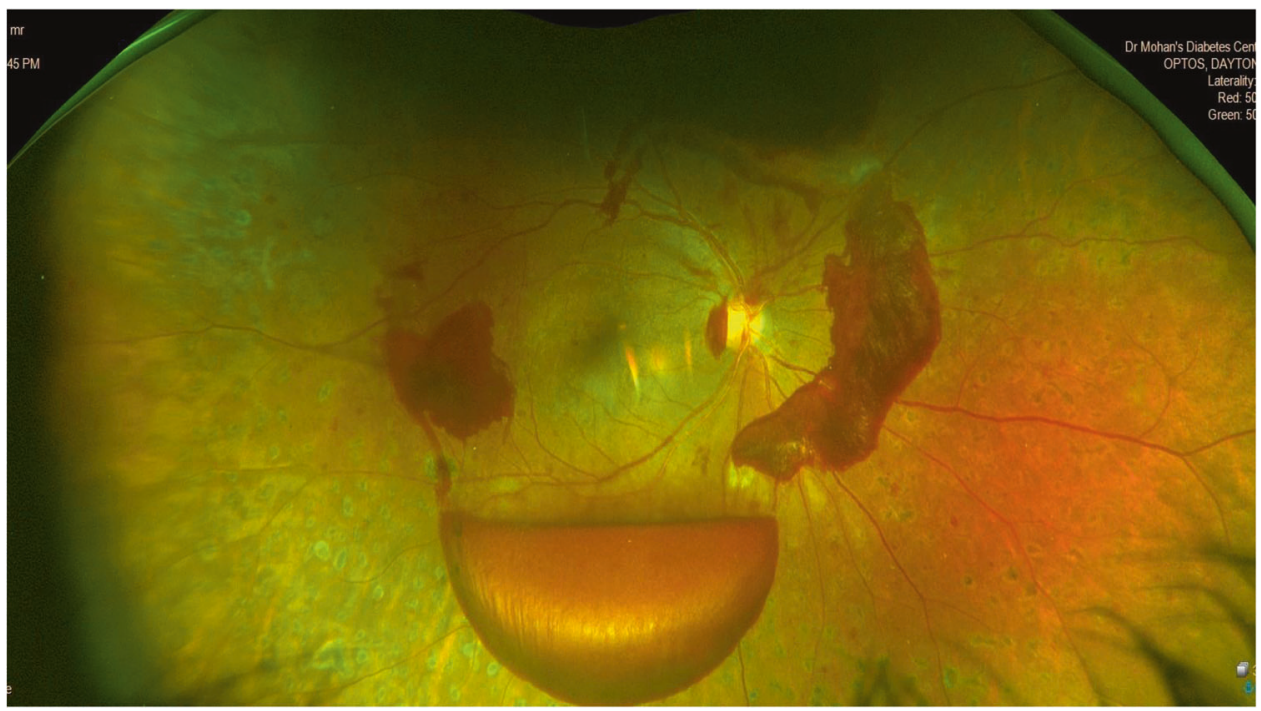

\section{Compliance with ethical standards}

Conflict of interest The authors declare that they have no conflict of interest.

Publisher's note Springer Nature remains neutral with regard to jurisdictional claims in published maps and institutional affiliations.

\section{References}

1. International Diabetes Federation. Diabetes Atlas, 9th ed. Brussels, Belgium: International Diabetes Federation; 2019. http://www.diabetesatlas.org. Accessed 14 Jun 2020.

2. Yau JW, Rogers SL, Kawasaki R, Lamoureux EL, Kowalski JW, Bek T, et al. Meta-analysis for eye disease (META-EYE) Study Group. Global prevalence and major risk factors of diabetic retinopathy. Diabetes Care. 2012;35:556-64.

3. Scanlon PH. Update on screening for sight-threatening diabetic retinopathy. Ophthalmic Res. 2019;62:218-24.

4. Flaxman SR, Bourne RRA, Resnikoff S, Ackland P, BraithwaiteT, Cicinelli MV, et al. Vision loss expert group of the Global Burden of Disease Study. Global causes of blindness and distance vision impairment 1990-2020: a systematic review and metaanalysis. Lancet Glob Health. 2017;5:e1221-34.

5. Piyasena MM, Murthy GV, Yip JL, Gilbert C, Peto T, Gordon I, et al. Systematic review and meta-analysis of diagnostic accuracy of detection of any level of diabetic retinopathy using digital retinal imaging. Syst Rev. 2018;7:182.

6. Dutt S, Sivaraman A, Savoy F, Rajalakshmi R. Insights into the growing popularity of artificial intelligence in ophthalmology. Indian J Ophthalmol. 2020;68:1339-46.

7. Grading diabetic retinopathy from stereoscopic color fundus photographs-an extension of the modified Airlie House classification. ETDRS report number 10. Early Treatment Diabetic Retinopathy Study Research Group. Ophthalmology. 1991;98:786-806.

8. Vujosevic S, Benetti E, Massignan F, Pilotto E, Varano M, Cavarzeran F, et al. Screening for diabetic retinopathy: 1 and 3 nonmydriatic 45-degree digital fundus photographs vs 7 standard Early Treatment Diabetic Retinopathy Study fields. Am J Ophthalmol. 2009;148:111-8.
9. Amit BJ, Jaya Prakash V, Muna B. Techniques of fundus imaging. Sci J Med Vis Res Foun. 2015;XXXIII:100-7.

10. Scanlon PH, Malhotra R, Greenwood RH, Aldington SJ, Foy C, Flatman $\mathrm{M}$, et al. Comparison of two reference standards in validating two field mydriatic digital photography as a method of screening for diabetic retinopathy. $\mathrm{Br} \mathrm{J}$ Ophthalmol. 2003;87:1258-63.

11. Aptel F, Denis P, Rouberol F, Thivolet C. Screening of diabetic retinopathy: effect of field number and mydriasis on sensitivity and specificity of digital fundus photography. Diabetes Metab. 2008;34:290-3.

12. Goh JK, Cheung CY, Sim SS, Tan PC, Tan GS, Wong TY, Retinal imaging techniques for diabetic retinopathy screening. J Diabetes Sci Technol. 2016;10:282-94.

13. Pradeepa R, Rajalakshmi R, Mohan V. Use of Telemedicine Technologies in diabetes prevention and control in resourceconstrained settings: lessons learned from emerging economies. Diabetes Technol Ther. 2019;21:S29-216.

14. Li HK, Horton M, Bursell SE, Cavallerano J, Zimmer-Galler I, Tennant $M$, et al. Telehealth practice recommendations for diabetic retinopathy, second edition. Telemed J E Health. 2011;17:814-37.

15. Panwar N, Huang P, Lee J, Keane PA, Chuan TS, Richhariya A, et al. Fundus photography in the 21st century-a review of recent technological advances and their implications for worldwide healthcare. Telemed J E Health. 2016;22:198-208.

16. Surendran TS, Raman R. Teleophthalmology in diabetic retinopathy. J Diabetes Sci Technol. 2014;8:262-6.

17. Mohan V, Prathiba V, Pradeepa R. Tele-diabetology to screen for diabetes and associated complications in rural India: The Chunampet rural diabetes prevention project model. J Diabetes Sci Technol. 2014;8:256-61.

18. Mohan R, Kohner EM, Aldington SJ, Nijhar I, Mohan V, Mather HM. Evaluation of a non-mydriatic camera in Indian and European diabetic patients. Br J Ophthalmol. 1988;72:841-5.

19. Taylor R, Lovelock L, Tunbridge WM, Alberti KG, Brackenridge RG, Stephenson P, et al. Comparison of non-mydriatic retinal photography with ophthalmoscopy in 2159 patients: mobile retinal camera study. BMJ. 1990;301:1243-7.

20. Boucher MC, Gresset JA, Angioi K, Olivier S. Effectiveness and safety of screening for diabetic retinopathy with two nonmydriatic digital images compared with the seven standard stereoscopic photographic fields. Can J Ophthalmol. 2003;38:557-68. 
21. Williams GA, Scott IU, Haller JA, Maguire AM, Marcus D, McDonald HR. Single-field fundus photography for diabetic retinopathy screening: a report by the American Academy of Ophthalmology. Ophthalmology. 2004;111:1055-62.

22. Gupta V, Bansal R, Gupta A, Bhansali A. Sensitivity and specificity of nonmydriatic digital imaging in screening diabetic retinopathy in Indian eyes. Indian J Ophthalmol. 2014;62:851-6.

23. Cunha LP, Figueiredo EA, Araújo HP, Costa-Cunha LVF, Costa $\mathrm{CF}$, Neto JMC, et al. Non-mydriatic fundus retinography in screening for diabetic retinopathy: agreement between family physicians, general ophthalmologists, and a retinal specialist. Front Endocrinol. 2018;9:251.

24. Lin DY, Blumenkranz MS, Brothers RJ, Grosvenor DM. The sensitivity and specificity of single-field nonmydriatic monochromatic digital fundus photography with remote image interpretation for diabetic retinopathy screening: a comparison with ophthalmoscopy and standardized mydriatic color photography. Am J Ophthalmol. 2002;134:204-13.

25. Murgatroyd H, Ellingford A, Cox A, Binnie M, Ellis JD, MacEwen $\mathrm{CJ}$, et al. Effect of mydriasis and different field strategies on digital image screening of diabetic eye disease. $\mathrm{Br} \mathrm{J}$ Ophthalmol. 2004;88:920-4.

26. Fenner BJ, Wong RLM, Lam W, Tan GSW, Cheung GCM. Advances in retinal imaging and applications in diabetic retinopathy screening: a review. Ophthalmol Ther. 2018;7:333-46.

27. Tran KT, Mendel TA, Holbrook KL, Yates PA. Construction of an inexpensive, hand-held fundus camera through modification of a consumer "point-and-shoot" camera. Investig Ophthalmol Vis Sci. 2012;53:7600-7.

28. Piyasena MMPN, Yip JLY, MacLeod D, Kim M, Gudlavalleti VSM. Diagnostic test accuracy of diabetic retinopathy screening by physician graders using a hand-held non-mydriatic retinal camera at a tertiary level medical clinic. BMC Ophthalmol. 2019;19:89.

29. Yogesan K, Constable IJ, Barry CJ, Eikelboom RH, McAllister IL, Tay-Kearney ML. Telemedicine screening of diabetic retinopathy using a hand-held fundus camera. Telemed J. 2000;6:219-23.

30. Sivaprasad S, Raman R, Conroy D, Mohan V, Wittenberg R, Rajalakshmi R, et al. The ORNATE India Project: United Kingdom-India Research Collaboration to tackle visual impairment due to diabetic retinopathy. Eye. 2020;34:1279-86.

31. Gosheva M, Klameth C, Norrenberg L, Clin L, Dietter J, Haq W, et al. Quality and learning curve of handheld versus stand-alone non-mydriatic cameras. Clin Ophthalmol. 2017;11:1601-6.

32. Davila JR, Sengupta SS, Niziol LM, Sindal MD, Besirli CG, Upadhyaya S, et al. Predictors of photographic quality with a handheld nonmydriatic fundus camera used for screening of visionthreatening diabetic retinopathy. Ophthalmologica. 2017;238:89-99.

33. Zhang W, Nicholas P, Schuman SG, Allingham MJ, Faridi A, Suthar T, et al. Screening for diabetic retinopathy using a portable, noncontact, nonmydriatic handheld retinal camera. J Diabetes Sci Technol. 2017;11:128-34.

34. Cuadros J, Bresnick G. Can commercially available handheld retinal cameras effectively screen diabetic retinopathy? J Diabetes Sci Technol. 2017;11:135-7.

35. Quellec G, Bazin L, Cazuguel G, Delafoy I, Cochener B, Lamard M. Suitability of a low-cost, handheld, nonmydriatic retinograph for diabetic retinopathy diagnosis. Transl Vis Sci Technol. 2016;5:16.

36. Ryan ME, Rajalakshmi R, Prathiba V, Anjana RM, Ranjani H, Narayan KM, et al. Comparison Among Methods of Retinopathy Assessment (CAMRA) Study: smartphone, nonmydriatic and mydriatic photography. Ophthalmology. 2015;122:2038-43.

37. Russo A, Morescalchi F, Costagliola C, Delcassi L, Semeraro F. Comparison of smartphone ophthalmoscopy with slit-lamp biomicroscopy for grading diabetic retinopathy. Am J Ophthalmol. 2015;159:360-4

38. Wintergerst MWM, Brinkmann CK, Holz FG, Finger RP. Undilated versus dilated monoscopic smartphone-based fundus photography for optic nerve head evaluation. Sci Rep. 2018;8:10228.

39. Bastawrous A, Giardini ME, Bolster NM, Peto T, Shah N, Livingstone IA, et al. Clinical validation of a smartphone-based adapter for optic disc imaging in Kenya. JAMA Ophthalmol. 2016;134:151-8.

40. Rajalakshmi R, Arulmalar S, Usha M, Prathiba V, Kareemuddin KS, Anjana RM, et al. Validation of smartphone based retinal photography for diabetic retinopathy screening. PLoS One. 2015:24;10.

41. Sengupta S, Sindal MD, Baskaran P, Pan U, Venkatesh R. Sensitivity and specificity of smartphone-based retinal imaging for diabetic retinopathy: a comparative study. Ophthalmol Retin. 2019;3:146-153.

42. Prathiba V, Rajalakshmi R, Arulmalar S, Usha M, Subhashini R, Gilbert CE, et al. Accuracy of the smartphone-based nonmydriatic retinal camera in the detection of sight-threatening diabetic retinopathy. Indian J Ophthalmol. 2020;68:S42-6.

43. Rajalakshmi R, Subashini R, Anjana RM, Mohan V. Automated diabetic retinopathy detection in smartphonebased fundus photography using artificial intelligence. Eye. 2018;32:1138-44.

44. Natarajan S, Jain A, Krishnan R, Rogye A, Sivaprasad S. Diagnostic accuracy of community-based diabetic retinopathy screening with an offline artificial intelligence system on a smartphone. JAMA Ophthalmol. 2019;137:1182-8.

45. Kim TN, Myers F, Reber C, Kim TN, Myers F, Reber C, et al. A smartphone-based tool for rapid, portable, and automated widefield retinal imaging. Transl Vis Sci Technol. 2018;7:21.

46. Sharma A, Subramaniam SD, Ramachandran KI, Lakshmikanthan C, Krishna S, Sundaramoorthy SK. Smartphone-based fundus camera device (MII Ret Cam) and technique with ability to image peripheral retina. Eur J Ophthalmol. 2016;26:142-4.

47. Staurenghi G, Viola F, Mainster MA, Graham RD, Harrington PG. Scanning laser ophthalmoscopy and angiography with a wide-field contact lens system. Arch Ophthalmol. 2005;123:244-52.

48. Silva PS, Cavallerano JD, Sun JK, Noble J, Aiello LM, Aiello LP. Nonmydriatic ultrawide field retinal imaging compared with dilated standard 7 -field $35-\mathrm{mm}$ photography and retinal specialist examination for evaluation of diabetic retinopathy. Am J Ophthalmol. 2012;154:549-59.e2.

49. Silva PS, Cavallerano JD, Sun JK, Soliman AZ, Aiello LM, Aiello LP. Peripheral lesions identified by mydriatic ultrawide field imaging: distribution and potential impact on diabetic retinopathy severity. Ophthalmology. 2013;120:2587-95.

50. Silva PS, Cavallerano JD, Haddad NM, Kwak H, Dyer KH, Omar $\mathrm{AF}$, et al. Peripheral lesions identified on ultrawide field imaging predict increased risk of diabetic retinopathy progression over 4 years. Ophthalmology. 2015;122:949-56.

51. Silva PS, Cavallerano JD, Tolls D, Omar A, Thakore K, Patel B, et al. Potential efficiency benefits of nonmydriatic ultrawide field retinal imaging in an ocular telehealth diabetic retinopathy program. Diabetes Care. 2014;37:50-5.

52. McBain VA, Forrester JV, Lois N. Fundus autofluorescence in the diagnosis of cystoid macular oedema. $\mathrm{Br} \mathrm{J}$ Ophthalmol. 2008;92:946-9.

53. Cicinelli MV, Cavalleri M, Brambati M, Lattanzio R, Bandello F. New imaging systems in diabetic retinopathy. Acta Diabetol. 2019;56:981-94.

54. Saurabh K, Roy R, Goel S. Correlation of multicolor images and conventional color fundus photographs with foveal autofluorescence patterns in diabetic macular edema. Indian J Ophthalmol. 2020;68:141-4. 